\title{
Numerical analysis of a railway compartment fire.
}

\author{
ENBAYA, A., ASIM, T., MISHRA, R. and RAO, R.B.K.N.
}

The final authenticated version is available online at: https://.doi.org/10.1007/978-3-319-23597-4 34. This pre-copyedited version is made available under the Springer terms of reuse for AAMs:

https://www.springer.com/gp/open-access/publication-policies/aam-terms-of-use. 


\section{Numerical Analysis of a Railway Compartment Fire}

\author{
Anwar Enbaya \\ University of Huddersfield \\ Huddersfield, UK \\ $\mathrm{HD} 13 \mathrm{DH}$ \\ (+44) 1484471027 \\ U0975950@hud.ac.uk
}

\author{
Taimoor Asim \\ University of Huddersfield \\ Huddersfield, UK \\ HD1 3DH \\ (+44) 1484472323 \\ t.asim@hud.ac.uk
}

\author{
Rakesh Mishra \\ University of Huddersfield \\ Huddersfield, UK \\ HD1 3DH \\ (+44) 1484473263 \\ r.mishra@hud.ac.uk
}

\author{
Raj B. K. N. Rao \\ COMADEM International \\ Birmingham, UK \\ B29 6DA \\ rajbknrao@btinternet.com
}

\begin{abstract}
Trains are considered to be the safest on-land transportation means for both passengers and cargo. Train accidents have been mainly disastrous, especially in case of fire, where the consequences are extensive loss of life and goods. The fire would generate smoke and heat which would spread quickly inside the railway compartments. Both heat and smoke are the primary reasons of casualties in a train. This study has been carried out to perform numerical analysis of fire characteristics in a railway compartment using commercial Computational Fluid Dynamics code ANSYS. Non-premixed combustion model has been used to simulate a fire scenario within a railway compartment, while Shear Stress Transport k- $\omega$ turbulence model has been used to accurately predict the hot air turbulence parameters within the compartment. The walls of the compartment have been modelled as no-slip stationary adiabatic walls, as is observed in real life conditions. Carbon dioxide concentration $\left(\mathrm{CO}_{2}\right)$, temperature distribution and air flow velocity within the railway compartment has been monitored. It has been observed that the smoke above the fire source flows to both sides of the compartment. The highest temperature zone is located downstream the fire source, and gradually decreased with the increase in the distance from the fire source. Hence, CFD can be used as an effective tool in order to analyse the evolution of fire in railway compartments with reasonable accuracy. The paper also briefly discusses the topical reliability issues.
\end{abstract}

\section{Keywords}

Computational Fluid Dynamics (CFD), ANSYS, Railway Compartment, Fire Modelling, $\mathrm{CO}_{2}$ Concentration.

\section{INTRODUCTION}

In the event of a train fire, the fire itself does not present the first danger to passengers. Instead, smoke from the fire is the primary danger. The inhalation of smoke causes the majority of firerelated injuries due to its emission of toxic gases. Smoke in the air can make it difficult for passengers to see exit doors clearly. Consequently, train passengers would be at serious risk of severe injury or even death, if the fire and smoke are allowed to become worse. It can be difficult to deal with train fires effectively, especially if there are many passengers on board [1]. The present study explores the flow and generation mechanism of temperature distribution and smoke in train fires.

A successful fire safety design can save lives. It is stated that large train fires can have severe consequences [2]. Cost is a primary consideration when designing a fire safety strategy. Fire size and occurrence can be lowered with higher levels of knowledge about the fire. It has been argued that designers are unable to fully estimate fires and they do not have enough knowledge of the ways in which fires behaves in the context of trains [3]. Air velocity is largely influenced by Heat Release Rate (HRR), which is generated from the train that has caught fire. Furthermore, the emergency tunnel ventilation system's performance is also controlled by this parameter. The type and amount of flammable materials within the train carriage, the characteristics of the train carriage (i.e. size, doors, windows, etc.), and the carriage construction type, determine HRR values [4]. HRR has the largest influence on how serious the fire becomes [5].

The aims of fire safety processes are to continuously improve and develop new systems that are responsive in case of fire emergencies. Currently, a new emergency response system is being explored to unite forecast and live sensor monitoring of fire development. The estimation of fire dynamics in the compartment is envisioned which will infer a paradigm change in the reaction to traumas by offering the fire service with vital evidence about the fire well ahead of time [6].

The precise forecast of the spread of smoke (poisonous gases) and distributions of temperature and velocity is vital for the scheme of detection of fire, and safety methods. It is also significant in offering testimony for the efficiency of precautionary actions, and controls the ventilation for smoke. The protocols for fire protection have to be employed while designing structures. In specific cases, for instance large public structures, museums, train stations, concert halls and tunnels, the fire protection protocols are very much significant in the case of emergency. Using these protocols, removal of the smoke 
generated is carried out using the instated devices throughout the time needed for the process of evacuation [7].

Modern fluid power systems are becoming complex and globally distributed. A number of issues are emerging which affect its reliability in one form or another. Some of these issues are common cause failures, reliability of computer codes and software, cyber security and risks, environmental safety issues, obsolescence issues, human factors such as behaviour, decision making, modelling and simulation issues, maintenance related issues etc. Reliability and robustness are critical and despite the advanced modelling and simulation techniques employed, many companies are finding that the operating environmental conditions are much harsher than predicted.

Uncertainties, Unreliability and Unavailability are closely related to each other and should be seriously treated as totally unacceptable by everyone right from the start. Historical evidences and plenty of literature exist to reveal the cost of unreliability and bad decision making in all walks of life. Its long-term consequences on health, wealth, quality of life and sustained prosperity of individuals and nations are recorded in histories of nations. Any number of multi-dimensional warranties, guarantees, laws and byelaws, etc. will never solve the problem of unreliability. Continuous awareness of the cost of unreliability and its dire short-term and long-term consequences should be effectively disseminated at all levels as a number one priority by all responsible people irrespective cast, creed, colour and religion. A number of case studies and bench marking studies exist that should be brought to light. It is time to initiate action research and action learning programs. Interdisciplinary research provides useful answers to many unanswered issues. Smart Integration, collaboration and proactive activities between industries, academia and professional organizations should be accelerated.

It is essential that effective systems for dealing with fire are created and employed in order to negate the risk of fire in passenger carriages, night carriages, restaurant carriages, power electronic parts and engine parts. Therefore, rail sectors require effective methods for investigating the spread of fire and the effectiveness of fire fighting strategies. However, the understanding of the evolution of fire in railway compartment needs to be understood in detail first, and hence Computational Fluid Dynamics (CFD) based analysis has been used in the present study to provide assistance to rail engineers when selecting the optimal fire fighting system setup [8,9]. Smoke's dispersion process in space and time, temperature and velocity variations and their effects on the train's emergency evacuation systems have been investigated in the present study.

\section{NUMERICAL MODELLING}

The numerical analysis of a fire in a railway compartment has been carried out with the aid of a commercial CFD code ANSYS. ANSYS code comprises of the physical models involving heat transfer, turbulent flows, chemical mixing, reacting flows, multiphase flows and combustion. Finite-volume method is used by ANSYS code to numerically solve the equations that govern a fluid [10]. The carbon dioxide concentration and temperature distribution in the railway compartment are being analysed in detail. Three dimensional partial differential equations for the conservation of energy; momentum and mass are iteratively solved over a time of 360 seconds, with a time step size of 1 second.

ANSYS's non-premixed combustion model has been implemented to simulate non-spreading fire in a railway compartment. Non-premixed combustion model consist of the solution of transport equations for one or two conserved scalars (the mixture fractions). Equations for individual species are not solved. Instead, species concentrations are derived from the predicted mixture fraction fields. The thermochemistry calculations are pre-processed and then tabulated for look-up in ANSYS. Interaction of turbulence and chemistry is accounted for with an assumed-shape Probability Density Function (PDF) $[11,12]$.

Combustion includes chemical reactions with the oxygen around it dragging air into the fire and generating hot gases that travel upwards. For a methane fire the reaction for complete combustion is:

$$
\mathrm{CH}_{4}+2 \mathrm{O}_{2} \rightarrow \mathrm{CO}_{2}+2 \mathrm{H}_{2} \mathrm{O}
$$

Incomplete combustion refers to a lack of air. However, in well ventilated conditions, the reaction follows the stoichiometry for complete combustion, so the quantity of carbon monoxide (CO) produced is negligible, hence is the case in present study.

\subsection{Geometry of the Flow Domain}

The dimensions of the computational domain of a railway compartment are $20 \mathrm{~m} \times 2.7 \mathrm{~m} \times 2.4 \mathrm{~m}$, which correspond to a conventional train compartment size in the UK, as shown in Figure 1. The fire has been numerically initiated in the centre of the compartment using a rectangular methane burner, having a surface area of $1 \mathrm{~m}^{2}$ and height of $0.4 \mathrm{~m}$. The fuel flow rate specified corresponds to a heat flux of $350 \mathrm{~kW}$. Ventilation is provided by two doors of $1.9 \mathrm{~m}$ height $\times 1.4 \mathrm{~m}$ width, one at each end, and assuming that the doors would open once the fire has been initiated.

To model the airflow through the open doors properly, and to minimize the effects of the boundaries on the fire development within the compartment, the outlet boundary of the computational domain has been extended outside both doors by $10 \mathrm{~m} \times 10 \mathrm{~m} \times 10 \mathrm{~m}$ to include a region outside the railway compartment.

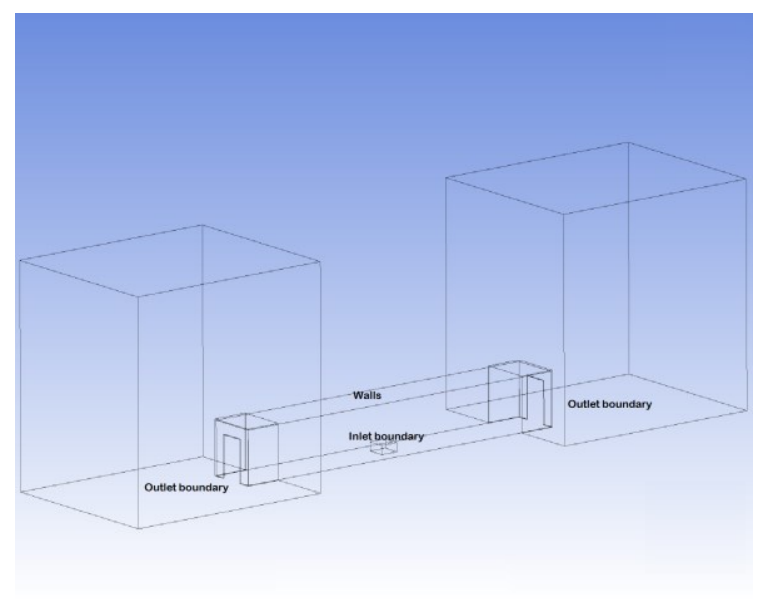

Figure 1. The geometry of the railway compartment. 


\subsection{Mesh Sensitivity Analysis}

In the CFD process, the quality of meshing plays a vital role. Hence, a mesh independent analysis has been performed to confirm the precision of the results, and to identify the most effective mesh sizing in order to achieve an appropriate mesh discretisation. The mesh has been created for the compartment using the Cutcell method. Figure 2 shows the meshing of the flow domain.

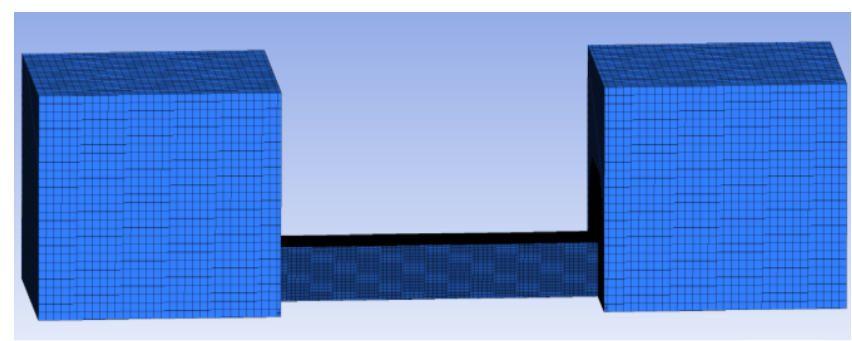

Figure 2. The mesh.

The numerical simulation is run using different mesh sizes. The first mesh comprises of 346,646 elements, the second mesh of 672,308 elements, and the third mesh of 709,906 elements. The average temperature distribution within the compartment from these three simulations is compared in Figure 3.

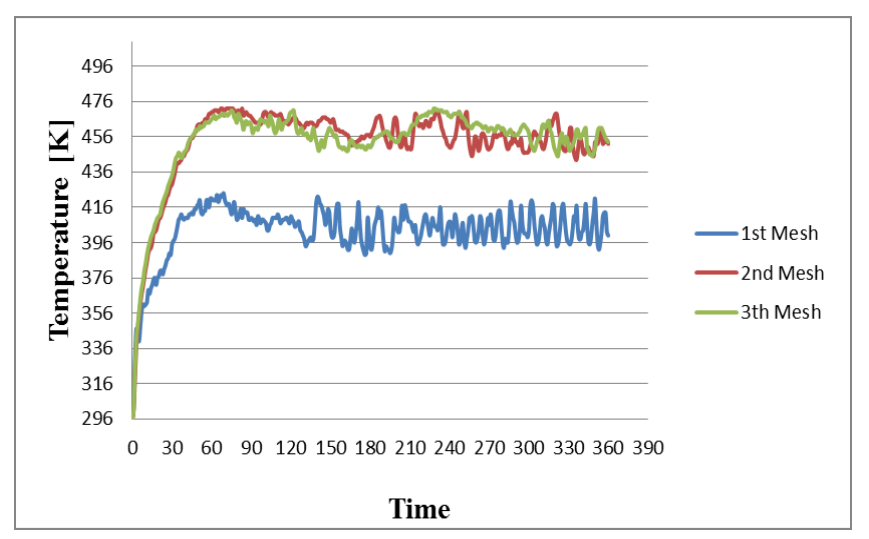

Figure 3. Mesh independence analysis.

It has been observed that the average temperature distribution within the compartment is well predicted by both the second and the third meshes, and the obtained results do not show significant changes. Furthermore, it is obvious that the simulation using the third mesh is less unstable. Therefore, this study employs the third mesh (comprising of 709,906 mesh elements) to investigate the air flow and temperature distribution within the railway compartment.

\subsection{Solver Setup}

Software Reliability is defined as: the probability of failure-free software operation for a specified period of time in a specified environment. Although Software Reliability is defined as a probabilistic function, and comes with the notion of time, we must note that unlike traditional Hardware Reliability, Software Reliability is not a direct function of time. Physical assets may will age with time and usage, but software will not age or wearout during its 'life cycle'. Software do fail due to many reasons such as: errors, ambiguities, oversights or misinterpretation of the specification that the software is supposed to satisfy, carelessness or incompetence in writing code, inadequate testing, incorrect or unexpected usage of the software or other unforeseen problems. Hardware faults are mostly physical faults, while software faults are (intentionally or unintentionally) human - induced design faults, which are much harder to visualize, classify, detect, and correct. Furthermore, design faults are closely related to fuzzy human factors, which we yet to fully understand. The quality and reliability of software will not change once it is uploaded and start running. Trying to achieve higher reliability by not knowing the root causes will hinder progress and is very costly.

Since the fire scenario is associated with chemical reactions, non-premixed combustion model has been implemented. The energy equation has been iteratively solved in order to predict the variations in the temperature. The solver settings and essential boundary conditions are summarised in Table 1 .

Table 1. Solver setup and boundary conditions.

\begin{tabular}{|c|c|}
\hline Parameter & Description \\
\hline Mode & Transient \\
\hline Solver & Pressure Based \\
\hline Turbulence Model & Shear Stress Transport k- $\omega$ \\
\hline Inlet & Mass Flow Inlet \\
\hline Outlet & Pressure Outlet \\
\hline Walls & Adiabatic - No slip \\
\hline
\end{tabular}

\section{RESULTS and DISCUSSION}

The temporal distribution of temperature within the railway compartment is depicted in Figure 4, where Figure 4(a) represents the scenario after 8seconds of fire eruption, while Figure 4(b) represents the scenario after 360 seconds of fire eruption within the compartment.

It can be seen that as the fire erupts within the railway compartment, because of its higher temperature (and lesser density), the hot air from the fire travels against the gravitational force, until it comes in contact with the ceiling of the compartment. Then it starts to spread outwards in both directions until it escapes out from the evacuation doors, and into the environment. It can be further seen in Figure 4(b) that the region of highest temperature $(742 \mathrm{~K})$ is on the ceiling, directly above the fire, while the temperature reduces as the distance from the source/fire increases. As there is a gap between the ceiling of the compartment and the evacuation doors, the hot air is trapped on the upper portions of the compartment, while the lower sections of the compartment are at comparatively lower temperature. 


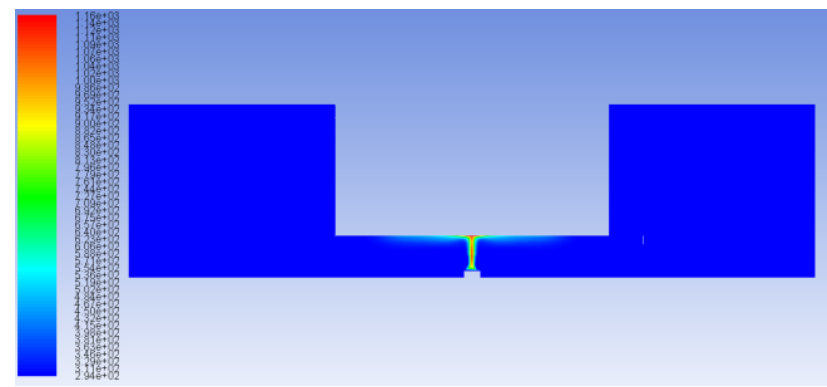

(a)

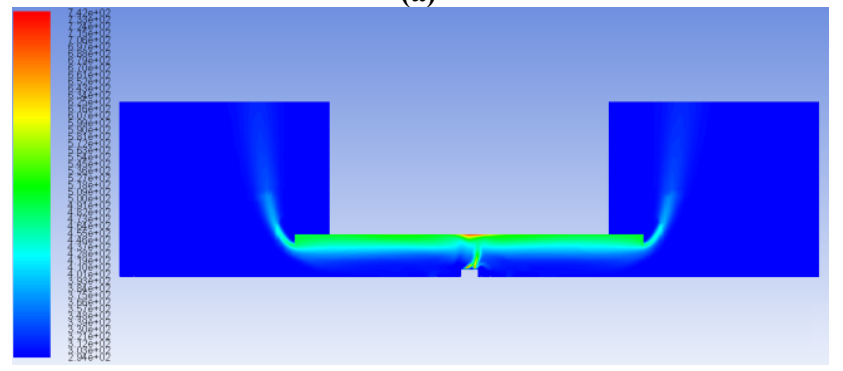

(b)

Figure 4. Static temperature variations after (a) $8 \mathrm{sec}$ and (b) 360 sec of fire eruption.

The evolution of fire can be clearly seen in the figures. Only a small region of the compartment is affected 8seconds after the fire has erupted. However, as the time increases, most of the compartment is filled with smoke, and hence most of the volume of the railway compartment is occupied by higher temperature zones.

Data is omnipresent. Data on its own is useless unless it is intelligently analysed and understood. It has been reported that the reliability of many research investigations carried out by many reputable organizations is coming under increasing scrutiny. It is also true that the entrenched culture of cut-throat competition and fraudulent behaviour is hindering the progress. All reliability investigations are heavily dependent upon the quality of data and the intelligent extracting capability of individuals. Extracting the intelligence from Big and Open Data is a challenging task indeed.

For further analysing the flow behaviour within the railway compartment, profiles of static temperature have been drawn on (a) a vertical line directly above the methane burner (Figure $5(\mathrm{a})$ ), and (b) a horizontal line in the middle of the compartment from one evaluation door to the other (Figure 5(b)). Figure 5(a) depicts that just above the methane burner, where the fire has erupted, the static temperature rises considerably; however, just after the fire, it drops back and then increases gradually while going towards the ceiling of the compartment. Furthermore, it can be seen that the fire is still pre-mature after 8 seconds of eruption.

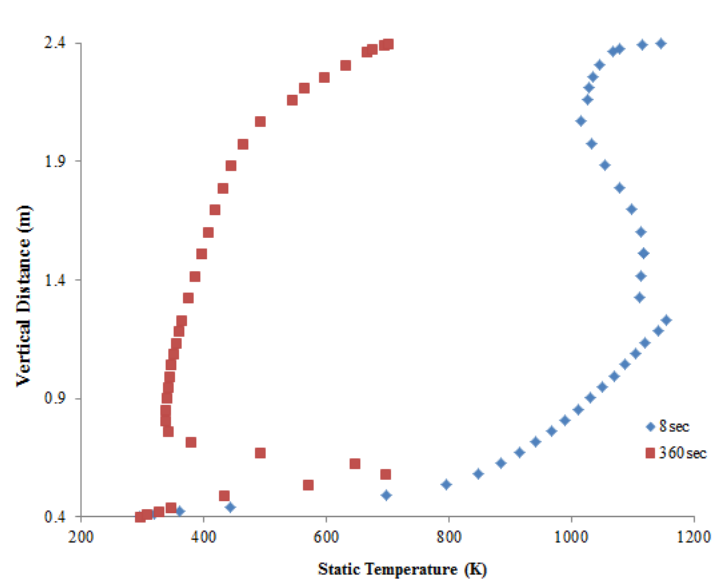

(a)

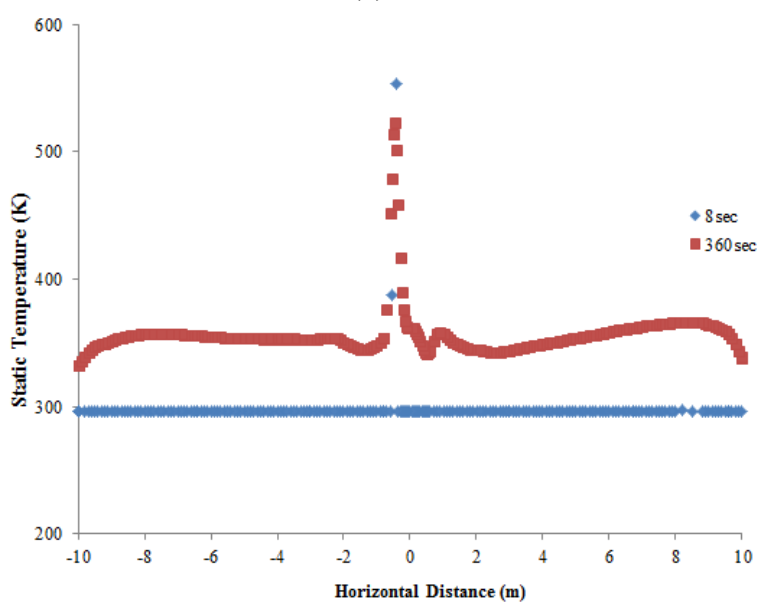

(b)

Figure 5. Static temperature profiles (a) above the methane burner and (b) along the length of the compartment.

Figure 5(b) depicts that after 8seconds of fire eruption, only the region directly above the fire is at higher temperature, whereas the rest of the compartment is at ambient temperature. However, after 360seconds, although the same trend follows but the ambient temperature within the compartment has increased significantly.

Further analysing the flow behaviour within the compartment, Figures 6(a) and (b) depict the variations in the flow velocity magnitude after 8 seconds and 360 seconds respectively. It can be seen that as the fire erupts, due to the higher temperature directly above the fire, the flow velocity increases, and then spreads outwards. This trend is similar to the one observed in case of temperature variations within the compartment at the same occasions. However, it should be noted that once enough time has elapsed, the flow velocity within the compartment reduces significantly, while the smoke is rushing out of the evacuation doors at significantly higher velocities. 


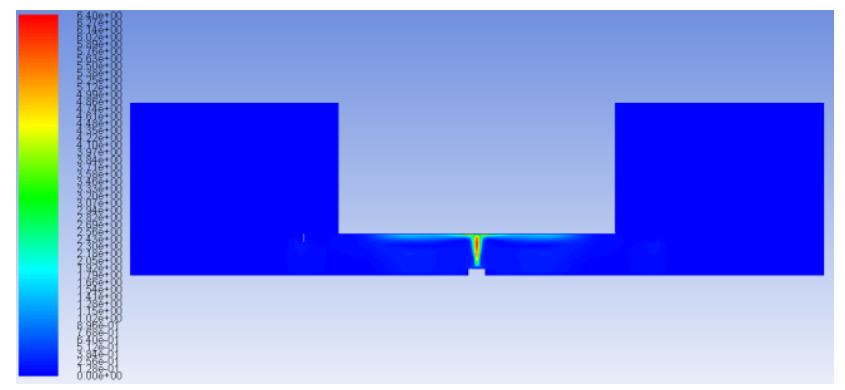

(a)

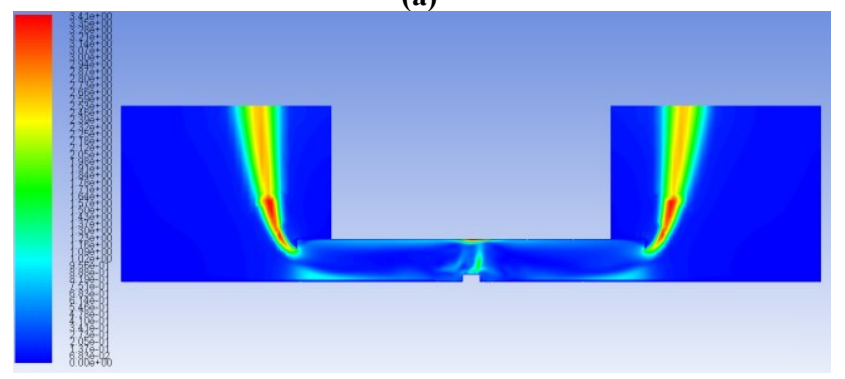

(b)

Figure 6. Velocity magnitude variations after (a) 8sec and (b) $360 \mathrm{sec}$ of fire eruption.

Figure 7 depicts the velocity vectors in the vicinity of the methane burner. It can be seen that the smoke from the fire travels upwards and then sideways to the evacuation doors, while, at the same time, fresh air is entering the compartment from the lower sections. It can be further noticed that the region in the centre of the compartment is almost stationary.

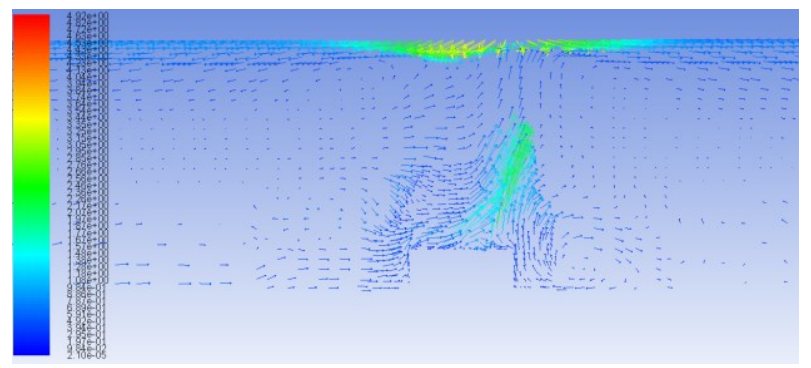

Figure 7. Velocity vectors in the vicinity of the methane burner after $360 \mathrm{sec}$ of fire eruption.

Figure 8 depicts the variations in the flow velocity magnitude at different time instants both vertically above the methane burner, and horizontally along the centre of the compartment. Figure 8(a) depicts that, after 8seconds of fire eruption, just above the methane burner, the flow velocity increases significantly, whereas the flow is stationary at the ceiling due to no-slip boundary condition. Furthermore, after 360 seconds of fire eruption, the flow velocity just above the methane burner is considerably higher. The flow than slows down until it reaches a certain height from where onwards, it starts spreading, and hence the flow velocity increases.

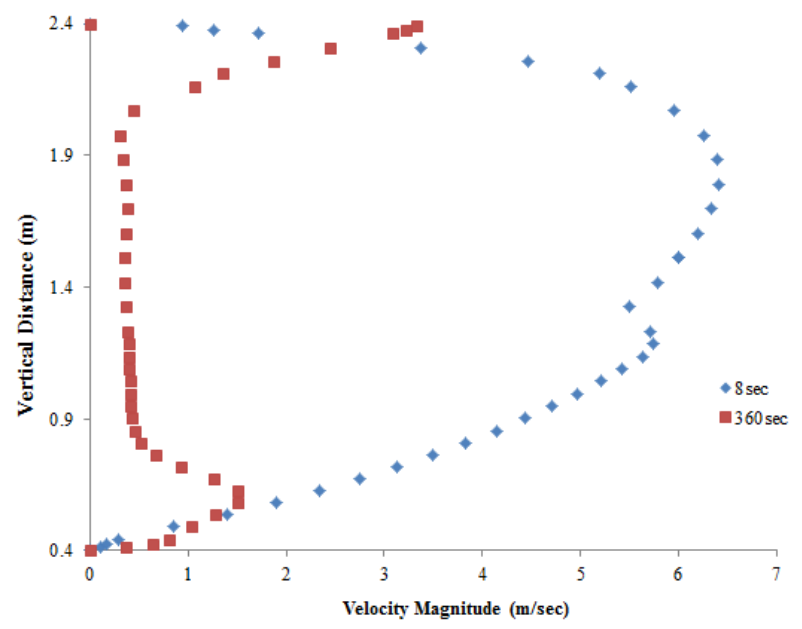

(a)

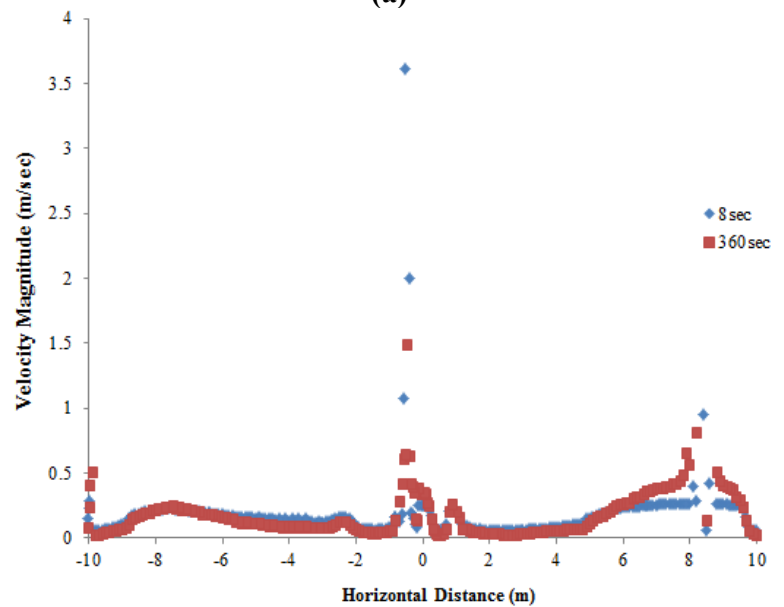

(b)

Figure 8. Velocity magnitude profiles (a) above the methane burner and (b) along the length of the compartment.

Figure 8 (b) depicts that only the region directly above the fire is at higher velocity. However, there is an indication that the flow again starts to accelerate near the evacuation doors. This has already been observed in Figure 6(b).

The temporal distribution of the molar concentration of $\mathrm{CO}_{2}$ within the railway compartment is depicted in Figure 9, where Figure 9(a) represents the scenario after 8seconds of fire eruption, while Figure 9(b) represents the scenario after 360 seconds of fire eruption within the compartment. It can be seen that as the fire erupts within the railway compartment, the smoke is generated, which, due to being lighter than air, travels against the gravitational force, until it comes in contact with the ceiling of the compartment. Then it starts to spread outwards in both directions until it escapes out from the evacuation doors, and into the environment. It can be further seen in Figure 9(b) that the smoke is trapped on the upper portions of the compartment, where its concentration is almost uniform, while the lower sections of the compartment are free of smoke. The evolution of smoke can be clearly seen in the figures. Only a small region of the compartment is filled with smoke 8seconds after the fire has erupted. However, as the time increases, most of the compartment is filled with smoke. 


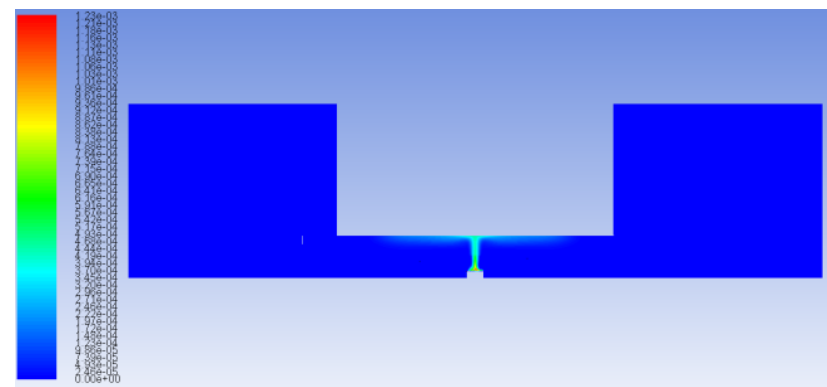

(a)

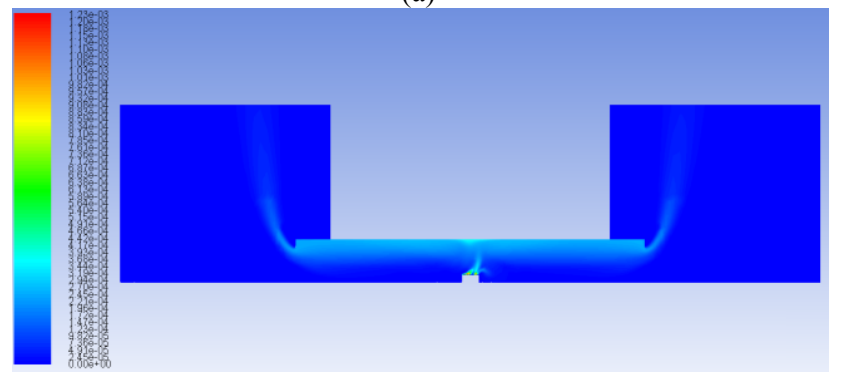

(b)

Figure 9. $\mathrm{CO}_{2}$ molar concentration variations after (a) $8 \mathrm{sec}$ and (b) 360sec of fire eruption.

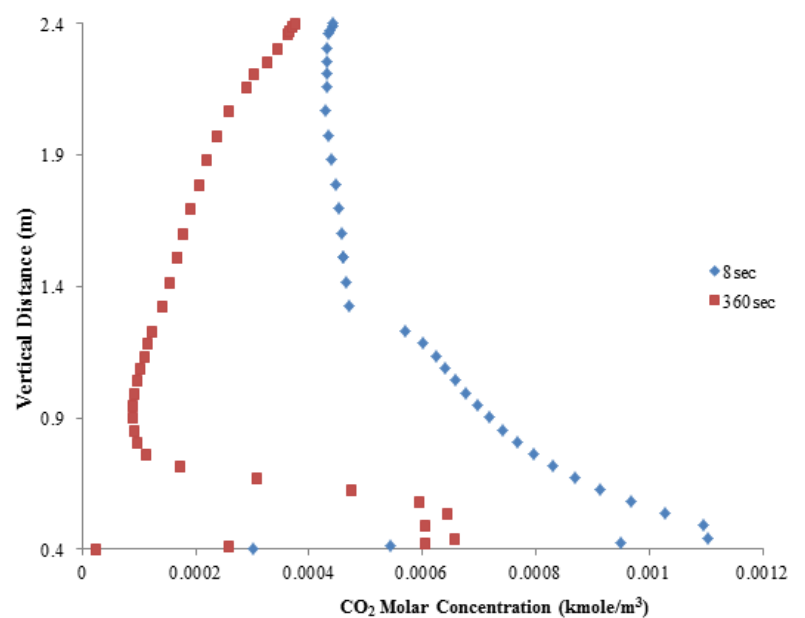

Figure 10. $\mathrm{CO}_{2}$ molar concentration profiles above the methane burner.

Figure 10 depicts the molar concentration of $\mathrm{CO}_{2}$ directly above the methane burner, both after 8 seconds and 360 seconds of fire eruption. It can be seen that in the early stages of fire, as it is still pre-mature, a lot of $\mathrm{CO}_{2}$ is being ejected into the compartment, although the overall region of the compartment occupied by the smoke is relatively small. However, after 360 seconds, the $\mathrm{CO}_{2}$ molar concentration is highest near the methane burner, which then increases gradually towards the ceiling of the compartment. $\mathrm{CO}_{2}$ molar concentration in the centre of the compartment, from one door to the other, has not been shown in the present study as this region is almost free of $\mathrm{CO}_{2}$ for the range of parameters considered in the present study. Hence, further studies need to be conducted in order to widen the range of $\mathrm{CO}_{2}$ concentration analysis.

\section{CONCLUSION}

Unavailability, poor quality, unreliable assets drive nations' invaluable resources to unsustainable and unrecoverable bottomless pit of misery and poverty of unimaginable dimension. Our 'Quality of life' and 'happiness' is heavily dependent upon reliable and sustainable performance of all assets under all operational conditions. Best practices, best guidelines and national/international standards are the only way to reduce uncertainties and enhance reliability of our assets through smart management practices.

A detailed CFD based investigations on a railway compartment fire has been carried out in the present study. Three primary parameters i.e. the static temperature, the flow velocity and the molar concentration of $\mathrm{CO}_{2}$, have been numerically analysed within a railway compartment. The spatio-temporal variations of these parameters indicate that as a fire erupts in a railway compartment, the smoke at higher temperature rises up and comes in contact with the ceiling of the compartment. Then it spreads outwards towards the evacuation doors on either ends of the compartment. The upper region of the compartment is filled with smoke, containing a large amount of $\mathrm{CO}_{2}$, while the lower section of the compartment is relatively free of smoke and $\mathrm{CO}_{2}$. It has further been noticed that the upper section of the railway compartment is at a higher temperature as compared to the lower section, and similarly the flow velocity is significantly higher in the top section of the compartment. Moreover, it has been observed that the smoke exits the compartment through the upper part of the evacuation doors, while fresh air enters the compartment from the lower part of these doors. Hence, CFD can be used as an effective tool in order to analyse railway compartment fires.

\section{REFERENCES}

[1] Mo, S., Li, Z., Liang, D., Li, J., \& Zhou, N. (2013). Analysis of Smoke Hazard in Train Compartment Fire Accidents Base on FDS. Procedia Engineering, 52,284289.

[2] White, N. (2010). Fire Development in Passenger Trains. Master Thesis. Centre for Environment Safety and Risk Engineering. Victoria University, Australia.

[3] Dowling, V. and White, N. (2004). Fire Sizes In Railway Passenger Saloons. Fire Safety Science, [online] 6, pp.6b3--1. Available at: http://www.iafss.org/publications/aofst/6/6b-3 [Accessed 30 Nov. 2014].

[4] Chiam, B.H. (2005). Numerical Simulation of a Metro Train Fire, Fire Engineering Research Report 05/1. Department of Civil Engineering. University of Canterbury. New Zealand.

[5] Babrauskas, V., \& Peacock, R. D. (1992). Heat release rate: The single most important variable in fire hazard. Fire Safety Journal, 18(3), 255-272.

[6] Jahn, W., Rein, G. \& Torero, J.L. (2009). The Effect of Model Parameters on the Simulation of Fire Dynamics. Fire Safety Science 9: 1341-1352.

[7] Rusch, D., Blum, L., Moser, A., \& Roesgen, T. (2008). Turbulence model validation for fire simulation by CFD 
and experimental investigation of a hot jet in crossflow. Fire Safety Journal, 43(6), 429-441.

[8] Andreini, A., Da Soghe, R., Giusti, A., \& Caruso, L. (2011). Pyrolysis Modeling and Numerical Simulation of Rail Carriage Fire Scenarios for the Safe Design of a Passenger Train. Department of Energy Engineering. University of Florence. Italy.

[9] Mishra, R., Singh, S. N., \& Seshadri, V. (1998). Study of wear characteristics and solid distribution in constant area and erosion-resistant long-radius pipe bends for the flow of multisized particulate slurries. Weir, 217(2), 297-306.

[10] Binbin, W. (2011). Comparative Research on FLUENT and FDS's Numerical Simulation of Smoke Spread in Subway Platform Fire. Procedia Engineering, 26, 1065-1075.

[11] ANSYS ${ }^{\circledR}$ Academic Research, Release 15.0, Help System, Theory Guide, ANSYS, Inc.

[12] Tesfa, B., Mishra, R., Gu, F., \& Ball, A. (2011). Combustion characteristics of $\mathrm{CI}$ engine running with biodiesel blends. International Conference on Renewable Energies and Power Quality, Las Palmas de Gran Canaria, Spain. 